\title{
EFEITOS POR COMPARAÇÕES E POR EXPERIMENTO EM INTERAÇÕES DE EXPERIMENTOS FATORIAIS
}

\author{
Comparisonwise and experimentwise effects in fatorial experiments interactions
}

\author{
Dilermando Perecin', Alberto Cargnelutti Filho²
}

\begin{abstract}
RESUMO
Avaliar efeitos de interações é um dos principais objetivos dos experimentos fatoriais. Em experimentos com dois fatores $A$ e $B$, com $m$ e $n$ níveis de cada fator, respectivamente, há $m \times n$ possíveis interações e $(m-1)(n-1)$ graus de liberdade associados. Freqüentemente somente parte dessas interações contribui efetivamente para a Soma de Quadrados da Interação e pode ser interessante examiná-las. O uso de nível de significância menos rigoroso para interpretação do efeito da interação "por experimento", em relação às demais fontes de variação da análise de variância, pode captar efeitos importantes. Recomenda-se o uso de $p=0,25$ para a interpretação do efeito da interação "por experimento", mantendo-se o usual p = 0,05 para efeitos "por comparações". Mesmo no caso de interações significativas, comparações selecionadas, em lugar de apenas "cortes", podem auxiliar a interpretação de interações complexas.
\end{abstract}

Termos para indexação: Análise de variância, desdobramento da interação, nível de significância.

\section{ABSTRACT}

To evaluate interaction is one of the most important objectives of fatorial experiments. In experiments with two factors $A$ and $B$, with $m$ and $n$ levels of each factor, there are $m \times n$ possible interactions and $(m-1)(n-1)$ degrees of freedom associated. Frequently just a part of these interactions contributes effectively to the sum of squares of interaction and must be interesting to evaluate them. The use of less rigorous level of significance for interpretation of the interaction "for experiment", in relation to sources of variation of the variance analysis, may catch important effect. One recommends the use of $p=0.25$ for the interpretation of the effect of the interaction "for experiment" remaining usual $p=0.05$ for effect "for comparisons". Even in the case of significant interactions, selected comparisons, instead of "cuts" only, may assist the interpretation of complex interactions.

Index terms: Analysis of variance, interaction sliced, significance level.

(Recebido em 14 de dezembro de 2006 e aprovado em 30 de maio de 2007)

\section{INTRODUÇÃO}

Um experimento é denominado fatorial quando cada tratamento combina simultaneamente níveis de dois ou mais fatores. Os valores qualitativos ou quantitativos que compõem cada fator são chamados níveis do fator e as combinações entre os níveis dos fatores formam os tratamentos do experimento fatorial (BARBIN, 2003; GOMES, 2000; STORCK et al., 2006).

Em experimentos fatoriais pode-se, por meio dos efeitos das interações, verificar se um fator é independente ou dependente do (s) outro (s). Se uma interação for não significativa evidencia que os fatores são independentes, ou seja, o comportamento de um fator independe da variação (ausência ou presença) de outro fator. Nesse caso as conclusões em separado para os fatores são válidas. No entanto, se uma interação for significativa indica que a resposta de um fator depende da presença ou ausência do outro. Nestes casos, uma das alternativas é estudar o comportamento de um fator dentro de cada nível de outro fator (BARBIN, 2003; GOMES, 2000; STORCK et al., 2006).

Uma das principais informações em experimentos fatoriais é a da interação entre os fatores, ou seja, verificar se as diferenças nas respostas dos níveis de um fator são similares ou diferentes em cada um dos níveis do (s) outro (s) fator (es). As interações são efeitos adicionais positivos (sinergismo) ou negativos (antagonismo) que aparecem quando se combinam níveis de dois ou mais fatores. No entanto, nem sempre é fácil de detectar ou analisar completamente os efeitos de interações.

O nível de significância do teste, ou seja, a máxima probabilidade de rejeitar a hipótese de nulidade $\left(\mathrm{H}_{0}\right)$ dado que ela é verdadeira, comumente usado em análises de

\footnotetext{
${ }^{1}$ Engenheiro Agrônomo, Doutor, Professor Titular - Departamento de Ciências Exatas - Faculdade de Ciências Agrárias e Veterinárias/FCAV Universidade Estadual Paulista/UNESP - Via de Acesso Professor Paulo Donato Castellane, s/n - 14884-900 - Jaboticabal, SP - perecin@fcav.unesp.br Bolsista do CNPq

${ }^{2}$ Engenheiro Agrônomo, Doutor, Professor - Departamento de Estatística - Universidade Federal do Rio Grande do Sul/UFRGS - Avenida Bento Gonçalves, 9500 - 91509-900 - Porto Alegre, RS - cargnelutti@ufrgs.br
} 
variância é 5\% ou 1\%. Caso não seja detectada interação por meio da análise de variância, procedimentos complementares dos efeitos principais dos fatores são realizados e o efeito da interação é desprezado (nulo). No entanto, em experimentos com dois fatores A e B, com m e $\mathrm{n}$ níveis de cada fator, respectivamente, há $\mathrm{m} \times \mathrm{n}$ possíveis efeitos de interações com (m-1)(n-1) graus de liberdade associados. Freqüentemente só parte dessas interações contribui efetivamente para a Soma de Quadrados da Interação e pode ser interessante examiná-las.

Pretende-se mostrar que o uso de nível de significância menos rigoroso, por experimento, pode ser útil para identificar efeitos importantes de algum nível de um fator dentro de algum nível de outro fator e/ou de combinações específicas que contribuem fortemente para a interação.

\section{MATERIAL E MÉTODOS}

Foram feitas considerações gerais sobre fatoriais ( 2 x 2) e sobre fatoriais (m x n). São apresentados exemplos com ilustrações hipotéticas para os fatoriais $(2 \times 2)$ e resultados de análises de dois experimentos fatoriais, conduzidos por pesquisadores, com a cultura da cana-deaçúcar.

O primeiro experimento fatorial $(3 \times 3)$ foi realizado pela Doutora Leila Luci Dinardo-Miranda do Centro de Cana do Instituto Agronômico de Campinas (IAC), visando avaliar a produtividade de cana-de-açúcar $\left(\mathrm{kg} \mathrm{parcela}^{-1}\right)$ submetida a três doses de um produto para controle de praga de solo em três épocas de aplicação. $\mathrm{O}$ experimento foi delineado em blocos ao acaso com seis repetições e com nove parcelas em cada bloco.

Um outro experimento de adubação de plantas de cana-de-açúcar com três doses de nitrogênio e três doses de fósforo foi extraído de Gomes (2000).

\section{RESULTADOS E DISCUSSÃO}

\section{Considerações gerais sobre fatoriais $(2 \times 2)$}

Seja um experimento fatorial A x B, com níveis $\mathrm{a}_{1}, \mathrm{a}_{2}$ e $b_{1}, b_{2}$, e para simplificar com $r$ repetições. Nesse caso, a interação tem apenas um grau de liberdade e seu efeito pode ser tomado pela diferença entre médias da diagonal e fora dela, ou seja, $\left(a_{1} b_{1}+a_{2} b_{2}\right)-\left(a_{1} b_{2}+a_{2} b_{1}\right)$.

Exemplo 1: Sejam as médias $a_{1} b_{1}=10, a_{2} b_{2}=10$, $a_{1} b_{2}=20$ e $a_{2} b_{1}=20$. Os efeitos principais de A e de B são nulos e há efeitos da interação (antagonismo). A análise mostra que a resposta do fator $\mathrm{A}$ ou do fator B depende do nível do outro fator. Para esse caso, uma maneira informativa é avaliar "cortes", ou seja, efeitos de um fator em cada nível do outro fator.
Exemplo 2: Sejam as médias $\mathrm{a}_{1} \mathrm{~b}_{1}=10, \mathrm{a}_{2} \mathrm{~b}_{2}=20$, $\mathrm{a}_{1} \mathrm{~b}_{2}=10$ e $\mathrm{a}_{2} \mathrm{~b}_{1}=10$. Há efeitos principais de $\mathrm{A}$ e de $\mathrm{B}$ e há interação A x B. A análise dos "cortes" é pouco informativa. Uma análise mais detalhada mostra que todos os efeitos aparecem exclusivamente pela combinação ou tratamento $a_{2} b_{2}$. Para esse caso uma análise informativa é a que leva em conta tratamento por tratamento (por exemplo, comparando-se médias por contrastes ou por comparações múltiplas).

\section{Considerações gerais sobre fatorial $\mathbf{m} \times \mathbf{n}$}

Seja um experimento fatorial A x B, com níveis m e n, respectivamente, para os fatores $\mathrm{A}$ e $\mathrm{B}$, e para simplificar com $r$ repetições. Nesse caso, a interação tem (m-1)(n-1) graus de liberdade. Quando se faz análise de variância "rotineira", a estatística F serve para testar a interação média ou "pooled". Pode-se dizer que é um teste da interação "por experimento". Há várias situações possíveis:

1) Interação significativa do tipo simples: As respostas de um fator não são similares para todos os níveis do outro fator: A interpretação pode ser obtida com 'cortes' da resposta de um fator para cada nível do outro fator. É um procedimento muito usual e eficaz para essa situação, detectando em quais 'cortes' ocorrem respostas diferentes que promovem interações significativas. $\mathrm{O}$ procedimento é denominado análise com desdobramento dos graus de liberdade.

2) Interação "quase significativa" do tipo simples: Mesmo nesse caso pode ser interessante examinar as interações mais detalhadamente. Podem ser construídos testes para examinar efeitos "por comparação". É uma situação com implicações teóricas similares as que ocorrem em outras áreas da estatística: Testes de coeficientes de regressão múltipla, procedimentos de comparações múltiplas, etc. Há taxas de erros associadas as interações do experimento como um todo e as associadas às comparações (O’NEILL \& WETHERILL, 1971). Algumas comparações podem ser muito mais importantes que outras (essa situação aparece no exemplo 3).

3) Interação significativa do tipo complexa: Diferentemente do caso anterior, as respostas responsáveis pelas interações não estão fortemente associadas a níveis de qualquer um dos fatores. Há $\mathrm{m}$ x $\mathrm{n}$ combinações ou tratamentos e as interações são devidas a tratamentos específicos. Para esses casos, a análise com auxílio de 'cortes' é pouco efetiva e a alternativa é avaliar todas "caselas" ou tratamentos, na tentativa de detectar melhores ou piores combinações dos níveis dos fatores. Essa situação é ilustrada no exemplo 4. 
Exemplo 3: Um experimento fatorial (3x3) foi realizado pela Doutora Leila Dinardo-Miranda do Centro de Cana do Instituto Agronômico de Campinas (IAC) visando a avaliar a produtividade de cana-de-açúcar $\left(\mathrm{kg}_{\text {parcela }}{ }^{-1}\right)$ submetida a três doses de um produto para controle de praga de solo em três épocas de aplicação. $\mathrm{O}$ experimento foi delineado em blocos ao acaso com seis repetições e com nove parcelas em cada bloco. O resumo da análise de variância, processada usando o SAS Institute (2004), encontra-se na Tabela 1.

A interação "por experimento" época x dose é significativa só a 8,62\% ( $\mathrm{p}=0,0862$, Tabela 1). Supondo o nível de significância de $5 \%$, comumente utilizado em análises de variância, evidenciaria que o efeito de interação é nulo. Interpretando dessa forma, a verificação da significância de efeitos principais de época e dose é um procedimento adequado, e neste caso evidencia efeito significativo somente de época de aplicação $(p=0,0104)$. Ao desdobrar a interação época $x$ dose, o efeito significativo de doses dentro de época $1(\mathrm{p}=0,0084)$ é visualizado. Portanto, se a análise não for explorada "por comparações", informações valiosas podem ser perdidas.

A significância do efeito de doses, somente na época 1, comprova uma hipótese inicial, fundamentada na forma sistêmica de ação do produto, ou seja, os efeitos de doses só se manifestam se o produto for aplicado na primeira época (início de desenvolvimento da cultura). As médias de produtividade por época e por dose e a significância do efeito de doses, somente na época 1 , podem ser vistas na Tabela 2 .

Este exemplo, entre tantos outros, demonstra a importância de observar efeitos da interação "por comparações" e não somente "por experimento". Embora seja um assunto pouco discutido em textos de análise de experimentos, alguma informação suplementar pode ser obtida em Bancroft (1968). Assim, do ponto de vista de análises de dados, sugere-se que o pesquisador seja mais tolerante com a taxa de erro "por experimento" (que é um teste de hipótese para a interação média), aceitando nível de significância, por exemplo, de $25 \%$, para captar efeitos importantes de interação "por comparações", aceitando significância, por exemplo, com 5\%.

Exemplo 4: Em experimentos de adubação de plantas, as respostas podem estar associadas aos balanceamentos entre os nutrientes ou ao nutriente no mínimo, regidos pela famosa Lei de Liebig (KREUZ et al., 1995), podendo então originar interações do tipo complexa. Para ilustrar, considere os dados das Tabelas 3 e 4 de um exemplo da produtividade de cana-de-açúcar em função de nitrogênio $(\mathrm{N})$ e fósforo (P), adaptado de Gomes (2000).

Tabela 1 - Análise de variância em relação à produtividade de cana-de-açúcar $\left(\mathrm{kg}_{\text {parcela }}{ }^{-1}\right)$ e desdobramento da interação Época x Dose.

\begin{tabular}{lcrrc}
\hline Fontes de Variação & GL & Quadrado Médio & F & Pr $>$ F \\
\hline Bloco & 5 & $8.294,1$ & 1,61 & 0,1806 \\
Época & 2 & $26.496,3$ & 5,13 & 0,0104 \\
Dose & 2 & $7.314,4$ & 1,42 & 0,2545 \\
Época*Dose & 4 & $11.365,7$ & 2,20 & 0,0862 \\
$\quad$ Doses dentro Época 1 & 2 & $27.839,0$ & 5,39 & 0,0084 \\
$\quad$ Doses dentro Época 2 & 2 & 372,2 & 0,07 & 0,9306 \\
$\quad$ Doses dentro Época 3 & 2 & $1.834,7$ & 0,35 & 0,7031 \\
Resíduo & 40 & $5.163,5$ & & \\
\hline
\end{tabular}

Fonte: Centro de Cana IAC.

Tabela 2 - Média da produtividade de cana-de-açúcar $\left(\mathrm{kg}_{\text {parcela }}{ }^{-1}\right)$ em cada época e dose de um produto para controle praga de solo.

\begin{tabular}{lccc}
\hline & Dose 1 & Dose 2 & Dose 3 \\
\hline Época 1 Época 2 & $658,3 \mathrm{~b}$ & $710,0 \mathrm{ab}$ & $793,3 \mathrm{a}$ \\
Época 3 & 710,0 & 706,7 & 721,7 \\
\hline
\end{tabular}

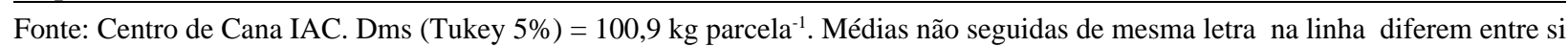
ao nível de $5 \%$ de probabilidade. 
Tabela 3 - Análise de variância da produtividade de cana-de-açúcar - dados em toneladas por hectare, extraído de Gomes (2000).

\begin{tabular}{lccrc}
\hline Fontes de Variação & GL & Quadrado Médio & F & Pr > F \\
\hline Nitrogênio (N) & 2 & 21,6 & 0,34 & 0,7148 \\
Fósforo (P) & 2 & $2.625,7$ & 42,06 & 0,0000 \\
Nitrogênio x Fósforo & 4 & 267,3 & 4,28 & 0,0094 \\
\hline 1) Cortes para níveis de P & & & & 0,80 \\
$\quad$ Nitrogênio dentro de P0 & 2 & 49,7 & 5,48 & 0,4628 \\
$\quad$ Nitrogênio dentro de P1 & 2 & 342,1 & 2,63 & 0,0110 \\
$\quad$ Nitrogênio dentro de P2 & 2 & 164,1 & & 0,0928 \\
\hline 2) Cortes para níveis de N & & & 22,86 & 0,0000 \\
$\quad$ Fósforo dentro de N0 & 2 & $1.427,2$ & 18,12 & 0,0000 \\
$\quad$ Fósforo dentro de N1 & 2 & $1.131,0$ & 9,64 & 0,0008 \\
$\quad$ Fósforo dentro de N2 & 2 & 602,1 & & \\
\hline Resíduo & 24 & 62,4 & & \\
\hline
\end{tabular}

Fonte: Adaptado de Gomes (2000).

Tabela 4 - Média da produtividade de cana-de-açúcar $\left(\mathrm{t} \mathrm{ha}^{-1}\right)$ de 6 parcelas para cada combinação das doses de nitrogênio $(\mathrm{N})$ e de fósforo $(\mathrm{P})$.

\begin{tabular}{lccc}
\hline & Dose 0 de N & Dose 1 de N & Dose 2 de N \\
\hline Dose 0 de P & $36,4 \mathrm{c}$ & $33,7 \mathrm{c}$ & $39,5 \mathrm{c}$ \\
Dose 1 de P & $44,2 \mathrm{bc}$ & $57,9 \mathrm{ab}$ & $56,5 \mathrm{ab}$ \\
Dose 2 de P & $66,1 \mathrm{a}$ & $57,0 \mathrm{ab}$ & $57,1 \mathrm{ab}$ \\
\hline
\end{tabular}

Fonte: Adaptado de Gomes (2000). Erro padrão das médias $=3,2 \mathrm{t} \mathrm{ha}^{-1}$. Dms (Tukey 5\%) =15,7 t ha-1 ${ }^{-1}$ Médias não seguidas de mesma letra diferem entre si ao nível de $5 \%$ de probabilidade (comparações entre as 9 médias).

A interação Nitrogênio x Fósforo é fortemente significativa ( $\mathrm{p}=0,0094)$, mas os "cortes" apresentados na Tabela 4 são pouco informativos. Somente os efeitos de N dentro das doses zero P0(ausência) e P2 são não significativos $(\mathrm{p}>0,05)$. Análises de comparações selecionadas, superfícies de respostas ou mesmo comparações múltiplas com todas as médias podem ser mais eficientes. A análise com comparações múltiplas (Tukey 5\%, Tabela 4) sugere que há um platô nas doses (1 e 1), (1 e 2), (2 e 1) e (2 e 2) de $\mathrm{N}$ e $\mathrm{P}$, respectivamente. Ou seja, as doses 1 de $\mathrm{N}$ e 1 de $\mathrm{P}$ provavelmente são satisfatórias. A resposta na dose 2 de $\mathrm{P}$ na ausência de $\mathrm{N}$ contribui fortemente para a complexidade da interação. Embora estranha, pode ser parcialmente explicada pelas funções do fósforo, com estímulo ao desenvolvimento inicial do sistema radicular e ampliação da zona de exploração, alterando o balanço dos nutrientes no solo (SILVA \& CASAGRANDE, 1983). Esse exemplo dá uma idéia da complexidade das interações e sugerem que comparações entre tratamentos ou "caselas" podem auxiliar na sua interpretação.

\section{CONCLUSÕES}

O uso de nível de significância menos rigoroso para interpretação do efeito da interação "por experimento", em relação às demais fontes de variação da análise de variância, pode captar efeitos importantes. Recomenda-se o uso de $\mathrm{p}=0,25$ para a interpretação do efeito da interação "por experimento", mantendo-se o usual $\mathrm{p}=0,05$ para efeitos "por comparações".

Mesmo no caso de interações significativas, comparações selecionadas ou múltiplas, em lugar de apenas “cortes", podem auxiliar a interpretação de interações complexas.

\section{REFERÊNCIAS BIBLIOGRÁFICAS}

BANCROFT, T. A. Topics in intermediate statistical methods. Ames: IOWA, 1968. v. 1, 129 p.

BARBIN, D. Planejamento e análise estatística de experimentos agronômicos. Arapongas: Midas, 2003. 208 p. 
GOMES, F. P. Curso de estatística experimental. 14. ed. Piracicaba: Nobel, 2000. 478 p.

KREUZ, C. L.; LANZER, E. A.; PARIS, Q. Funções de produção Von Liebig com rendimentos decrescentes. Pesquisa Agropecuária Brasileira, Brasília, v. 30, p. 95106, 1995.

O’NEILL, R.; WETHERILL, G. B. The present state of multiple comparison methods. Journal Royal Statistics Society B, [S.1.], v. 33, p. 218-250, 1971.
SAS INSTITUTE. SAS onlinedoc 9.1.3. Cary, 2004.

SILVA, L. C. F.; CASAGRANDE, J. C. Nutrição mineral da cana-de-açúcar (macronutrientes). In: ORLANDO FILHO, J. Nutrição e adubação da cana-de-açúcar no Brasil. Piracicaba: IAA/PLANALSUCAR, 1983. 369 p.

STORCK, L.; GARCIA, D. C.; LOPES, S. J.; ESTEFANEL, V. Experimentação vegetal. 2. ed. Santa Maria: UFSM, 2006. $198 \mathrm{p}$. 